\title{
BMJ Open Effect of water, sanitation and hygiene interventions alone and combined with nutrition on child growth in low and middle income countries: a systematic review and meta-analysis
}

\author{
Tolesa Bekele (D , , ${ }^{1,2}$ Patrick Rawstorne, ${ }^{1}$ Bayzidur Rahman ${ }^{1}$
}

To cite: Bekele T, Rawstorne P, Rahman B. Effect of water, sanitation and hygiene interventions alone and combined with nutrition on child growth in low and middle income countries: a systematic review and meta-analysis. BMJ Open 2020;10:e034812. doi:10.1136/ bmjopen-2019-034812

- Prepublication history and additional material for this paper are available online. To view these files, please visit the journal online (http://dx.doi. org/10.1136/bmjopen-2019034812).

Received 07 October 2019 Revised 27 February 2020 Accepted 20 May 2020

A) Check for updates

(C) Author(s) (or their employer(s)) 2020. Re-use permitted under CC BY-NC. No commercial re-use. See rights and permissions. Published by BMJ.

${ }^{1}$ School of Public Health and Community Medicine, University of New South Wales, Sydney, New South Wales, Australia ${ }^{2}$ Department of Public Health, College of Medicine and Health Sciences, Ambo University, Ambo, Oromia, Ethiopia

Correspondence to

Mr Tolesa Bekele;

t.okuba@student.unsw.edu.au

\section{ABSTRACT}

Objective This study aimed to provide clarification on the benefits of water, sanitation and hygiene (WASH) alone separately and combined with nutrition in improving child growth outcomes.

Design Systematic review and meta-analysis.

Methods We conducted a systematic review using the Preferred Reporting Items for Systematic Review and Meta-Analyses guidelines. PubMed, MEDLINE, EMBASE, Scopus, Cochrane Library, Web of Science and Science Direct were searched in May 2018 and last updated in April 2019. We included studies that reported WASH interventions alone separately or combined with nutrition. Fixed and random-effects models were used to estimate pooled effect in mean difference (MD). Heterogeneity and publication bias statistics were performed.

Results A total of 18 studies were included: 13 cluster randomised controlled trials (RCTs) and 5 non-randomised controlled trials (non-RCTs). Non-RCTs showed effect of WASH interventions alone on height-for-age z-score (HAZ) (MD $=0.14 ; 95 \% \mathrm{Cl} 0.08$ to 0.21 ) but RCTs did not. WASH alone of non-RCTs and RCTs that were delivered over 1860 months indicated an effect on $\mathrm{HAZ}$ (MD $=0.04 ; 95 \% \mathrm{Cl}$ 0.01 to 0.08 ). RCTs showed an effect for children $<2$ years ( $\mathrm{MD}=0.07 ; 95 \% \mathrm{Cl} 0.01$ to 0.13 ). Non-RCTs of WASH alone and those that included at least two components, improved HAZ (MD $=0.15 ; 95 \% \mathrm{Cl} 0.07$ to 0.23 ) but RCTs did not. WASH alone of non-RCTs and RCTs separately or together showed no effect on weight-for-age z-score (WAZ) and weight-for-height z-score (WHZ). Combined WASH with nutrition showed an effect on $\mathrm{HAZ}$ (MD=0.13; $95 \% \mathrm{Cl} 0.08$ to 0.17 ) and on $\mathrm{WAZ}$ (MD=0.09; $95 \% \mathrm{Cl} 0.05$ to 0.13 ) and was borderline on WHZ.

Conclusions WASH interventions alone improved HAZ when delivered over 18-60 months and for children $<2$ years. Combined WASH with nutrition showed a strong effect on HAZ and WAZ and a borderline effect on WHZ. Integrated WASH with nutrition interventions may be effective inimproving child growth outcomes.

\section{INTRODUCTION}

Child growth failure (CGF), which is known as under-5 stunting, wasting and being underweight, is a specific subset of child
Strengths and limitations of this study

- This study reviewed evidence from studies that were conducted in low-income and middle-income countries following the guidelines of Preferred Reporting Items for Systematic Review and Meta-Analyses.

- We explored data from both randomised controlled trials and non-randomised studies.

- Sources of heterogeneity were assessed using subgroup analyses and meta-regressions based on prior identified factors.

- As few studies were included in the meta-analysis, this study could not confirm that the effect of water, sanitation and hygiene (WASH) plus nutrition versus nutrition or WASH on child growth may be due to nutrition only.

- Most of the methodological quality of included studies was deemed very low, low and medium, that is, none of the included studies have low risk of bias.

undernutrition that excludes micronutrient deficiencies. ${ }^{1}$ Estimations of stunting, wasting and being underweight can serve as a comprehensive assessment of CGF. CGF (height-for-age z-score (HAZ), weight-for-age z-score (WAZ) and weight-for-height z-score (WHZ) below -2 SD) based on Child Growth Standards of the WHO begins in the early life of a child. ${ }^{23}$

Water, sanitation and hygiene (WASH) interventions refer to the entire suite of activities on water quality and quantity, sanitation, handwashing with soap, food and environmental hygiene. In 2015, an estimated 663 million people worldwide had no access to improved drinking water sources ${ }^{4}$ and 1.9 billion people relied on drinking water that was faecally contaminated. ${ }^{5}$ Similarly, 2.4 billion people, that is one-third of the world's population, were estimated to lack access to improved sanitation facilities ${ }^{6}$ 
and $13 \%$ practised open defecation. ${ }^{4}$ Sub-Saharan Africa and South Asia continue to have the lowest sanitation coverage. ${ }^{4}$ Freeman et al reported that only $19 \%$ of people around the world wash their hands with water and/or soap after contact with excreta. ${ }^{7}$

Recent evidence found there may be an effect of WASH interventions on $\mathrm{CGF}^{8}{ }^{9}$ However, the exact mechanism and evidence for how WASH interventions might improve child growth are still under consideration. ${ }^{2}$ Some studies have found no benefits of WASH interventions in improving child growth ${ }^{3}$ and in contrast some studies found small effects. ${ }^{10}{ }^{11}$ Combined WASH components have improved HAZ (mean difference $(\mathrm{MD})=0.22$ ) and reduced the risk of stunting (Relative Risk $(\mathrm{RR})=0.87) .{ }^{12}$ A previous study suggested that universal coverage (90\%) of nutrition interventions in areas with a high burden of CGF would only decrease stunting by $20 \% .{ }^{13}$ Inflammation caused by environmental enteric dysfunction due to inadequate WASH exposure ${ }^{14}$ may explain why nutritional interventions are often unsuccessful. ${ }^{15}{ }^{16}$ Current interventions are examining the effect of WASH alone and combined with nutrition on child growth outcomes. To date, few meta-analyses have been conducted to examine the effect of WASH alone.$^{11} 1217$ We have included recently published trials with well-designed methods in the current review. To our knowledge, there is no study which has reviewed the effect of combined WASH with nutrition interventions on child growth. The current review aims to review the effect of WASH interventions alone separately and when combined with nutrition on child growth.

\section{METHODS}

\section{Search strategy and screening}

In this review, we followed the procedures of the Preferred Reporting Items for Systematic Reviews and Meta-Analyses (PRISMA 2009 Checklist) statement (http://www.prisma-statement.org/) (online supplementary checklist 1). ${ }^{18}$ We used the following search terms: 'Child,' 'Infant,' 'Preschool,' 'Growth,' 'Stunting,' 'Wasting,' 'Underweight,' 'Undernourished,' 'Heightfor-age,' 'Water,' 'Sanitation,' 'Hygiene,' 'Handwashing,' and 'Water disinfection.' PubMed, MEDLINE, EMBASE, Scopus, Cochrane Library, Web of Science and Science Direct were searched for articles in May 2018 and last updated in April 2019 for new articles. Additional search engines Google and Google Scholar were searched for grey literature and $\mathrm{PhD}$ dissertations. Furthermore, we hand-searched citations of key literature to identify relevant articles. This review details the search strategy within the body of the review, while the detail of search terms is provided in the supplementary file (online supplementary table 1). The identified articles were exported to citation manager EndNote V.X8 reference software and duplicates were discarded.

Inclusion criteria included randomised controlled trials and non-RCT studies, interventions on WASH alone (individual or combined) or combined with nutrition, reported mean and SD or error for z-score of child growth outcomes in both arms, children under-5 years of age, written in English and no limit on year of publication. Reporting child growth and malnutrition using summary measure such as mean and SD z-score has an advantage over the cut-off-based prevalence (stunted, underweight and wasted) approach. ${ }^{19}$ As such, we have considered reports with mean HAZ, WAZ and WHZ only. Searched articles were screened in three stages-by going through the titles, then abstract and finally the full text. We used a PRISMA flow diagram (http://www.prisma-statement. $\operatorname{org} /)^{18}$ to present the results of identified articles.

\section{Data extraction and quality assessment}

The primary author (TB) conceived the idea, searched and screened all relevant articles, extracted the data, performed analysis, assessed quality and drafted the manuscript. PR and BR checked the screened and excluded studies by the primary author and edited the manuscript. Mutual consensus among authors for excluded studies was sought through discussion when disagreements arose between authors. Data extraction and assessments were performed using the Cochrane Effective Practice and Organization of Care (EPOC) (https://epoc.cochrane. org) ${ }^{20}$ approach. Quality evaluation criteria were categorised into two broad parts. Part I: general domains which comprised information such as study design, participant information, component of interventions and outcome measures with overall weighting of $40 \%$. Points were given for each item based on the level of adequate information and the overall score was added to the maximum of 100 points.

We assigned greater weight (60\%) for Part II (risk of bias reduction) which assessed detailed quality of the research methods. We used item questions which are described in Cochrane EPOC risk of bias tools ${ }^{20}$ for studies with separate control groups with few items added. About 10-item questions were used in the current study. All included studies were rated against each question as having either a low risk, unclear risk or high risk of bias. Scores were assigned for each item question from 0 to 10 points and the overall points add up to a maximum of 100 points. For an item, 10 points were allocated for adequate methods (low risk), 5 points for methods that were inadequate (unclear risk) and 0 if the methods were not reported (high risk). To judge the quality of each included study, a weighted mean ${ }^{2122}$ was calculated for each included study using scores as well as weights from both Part I and Part II. The overall quality was judged to be very low quality (weighted mean $(\mathrm{WM})=0-54.9)$, low quality $(\mathrm{WM}=55-64)$, moderate quality ( $\mathrm{WM}=65-74)$, good quality ( $\mathrm{WM}=75$ to $84)$ and high quality (WM=85-100).

\section{Data analysis and synthesis}

We stored and analysed data using the Cochrane Community Review Manager Software (RevMan V.5.3 for Windows). ${ }^{23}$ Pooled results were reported using mean difference with 
95\% CI as well as forest plots created using both fixed and random-effects models. Subgroup analyses were performed for WASH interventions alone based on age, length of interventions, geographical regions and types of interventions. Heterogeneity between studies was checked using Cochrane Q-test statistics and quantified using $I^{2}$ statistics (ie, $I^{2}=100 \%(\mathrm{Q}-\mathrm{DF}) / \mathrm{Q}$, where $\mathrm{Q}$ is Cochrane's $\chi^{2}$ test statistics and DF is degree of freedom) ${ }^{24}$ Heterogeneity was classified as $I^{2}: 0 \%-40 \%$ minimal, $30 \%-60 \%$ moderate, $50 \%-90 \%$ substantial and $90 \%-100 \%$ considerable. Metaregression and statistical tests for publication bias were performed by Stata V.11.2 SE.

We explored publication bias by checking asymmetry of funnel plots. Egger's test statistic was used to check significant publication bias. Sensitivity analysis was performed by removing each study step-by-step in forest plots as well as by checking whether fixed and random-effects models produced different pooled estimates. We separately added sources of heterogeneity through potential moderators and investigated their influence on the effect measures using meta-regression models.

\section{Patient and public involvement}

Patients and the public were not involved in the design or conduct of the study.

\section{RESULTS}

\section{Description of studies}

Of all searched 3561 articles, 1312 were removed due to duplication. Based on titles and abstracts of 2249 articles, 2186 were excluded after comparing titles and information reported in the abstracts against the inclusion criteria of the current review. The full text of 63 articles were assessed for eligibility which resulted in excluding 43 studies. We contacted four authors of the excluded studies on the basis that if requested data were provided, those studies would fulfil the criteria to be included in the review. However, none of the contacted authors responded to our request. The remaining 20 studies satisfied the inclusion criteria set for a study to be included in the current review. We contacted authors of seven included studies for additional information and we received responses from five authors. ${ }^{25-29}$ Finally, we included 18 studies for quantitative synthesis, of which 15 were peer reviewed and 3 were World Bank working reports. Based on the exclusion criteria, all included studies were conducted in low-income and middle-income countries.

Duration of interventions ranged from 6 to 60 months: 6 months $^{30-32}$; 12 months ${ }^{27} 2933$ 34; 18 months ${ }^{35-37} ; 21$ months $^{38} ; 24$ months $^{2639-41} ; 30$ months $^{28} ; 36$ months $^{42}$ and 60 months. ${ }^{25}{ }^{43}$ All interventions considered children $<5$ years of age. Interventions were provided based on the presence of under-5 years of age and/or pregnant women in the selected households and the sample size raged from $88^{31}$ to $4360^{38}$ (figure 1 ).

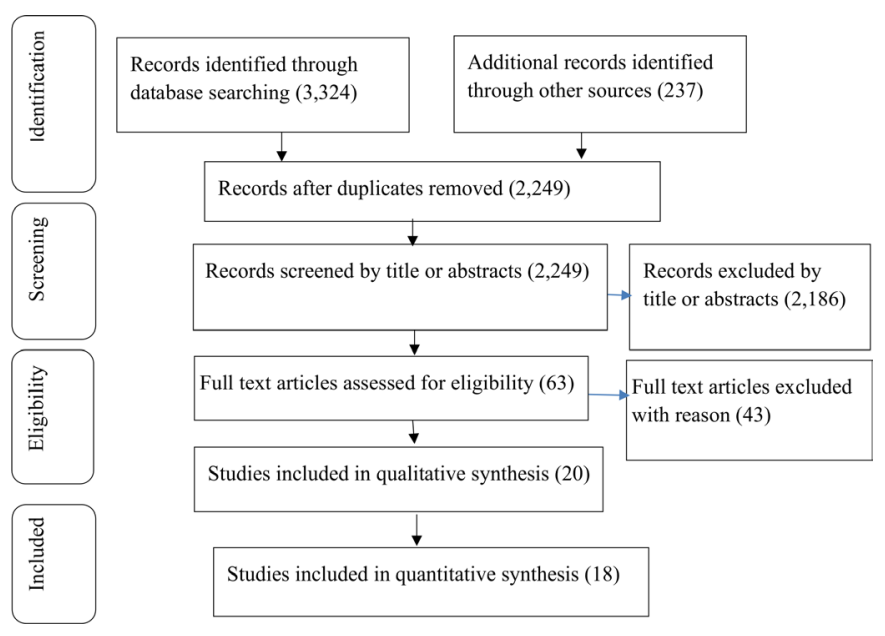

Figure 1 The Preferred Reporting Items for Systematic Review and Meta-Analyses flow diagram.

\section{Description of interventions and controls Interventions}

Included interventions were those with at least one component of WASH or combined with nutrition. WASH interventions aimed to improve water quality, water supply, improve sanitation and handwashing. Method of delivery could include behaviour change via education, such as total sanitation campaign and handwashing promotion, more direct interventions such as drinking water disinfection, latrine renovation or construction, or the combination of both behavioural change and direct interventions. Combined WASH with nutrition interventions included any of the above plus young child feeding practices.

\section{Controls}

Controls included study participants without any of the above interventions (ie, no water disinfection, continued to practise open defecation, no handwashing promotion and stations, no WASH behavioural change via education and no nutrition intervention programmes). The description of interventions for the included studies is summarised in table 1 .

\section{Height-for-age}

This section presents pooled estimates of WASH interventions alone regardless of the number of WASH components that were implemented. WASH interventions alone were shown to influence HAZ in the pooled effect estimate of non-RCTs (MD $=0.14 ; 95 \%$ CI 0.08 to 0.21 , $\mathrm{p}=0.001$ ). Of five non-RCTs, two ${ }^{39} 43$ showed an increased effect on HAZ (MD $=0.22 ; 95 \%$ CI 0.12 to 0.32 ) and ( $\mathrm{MD}=0.19 ; 95 \%$ CI 0.46 to 1.36 ) while the other three did not, ${ }^{25} 3142$ with substantial heterogeneity $\left(I^{2}=81 \%\right)$. In the RCTs, only one ${ }^{35}$ showed evidence of an effect on HAZ $(\mathrm{MD}=0.17 ; 95 \% \mathrm{CI} 0.07$ to 0.27$)$ and the pooled estimate was not significant (MD $=0.00 ; 95 \%$ CI -0.03 to 0.04 ), with moderate heterogeneity $\left(I^{2}=51 \%\right)$. The overall estimate of non-RCT and RCT studies together showed no 


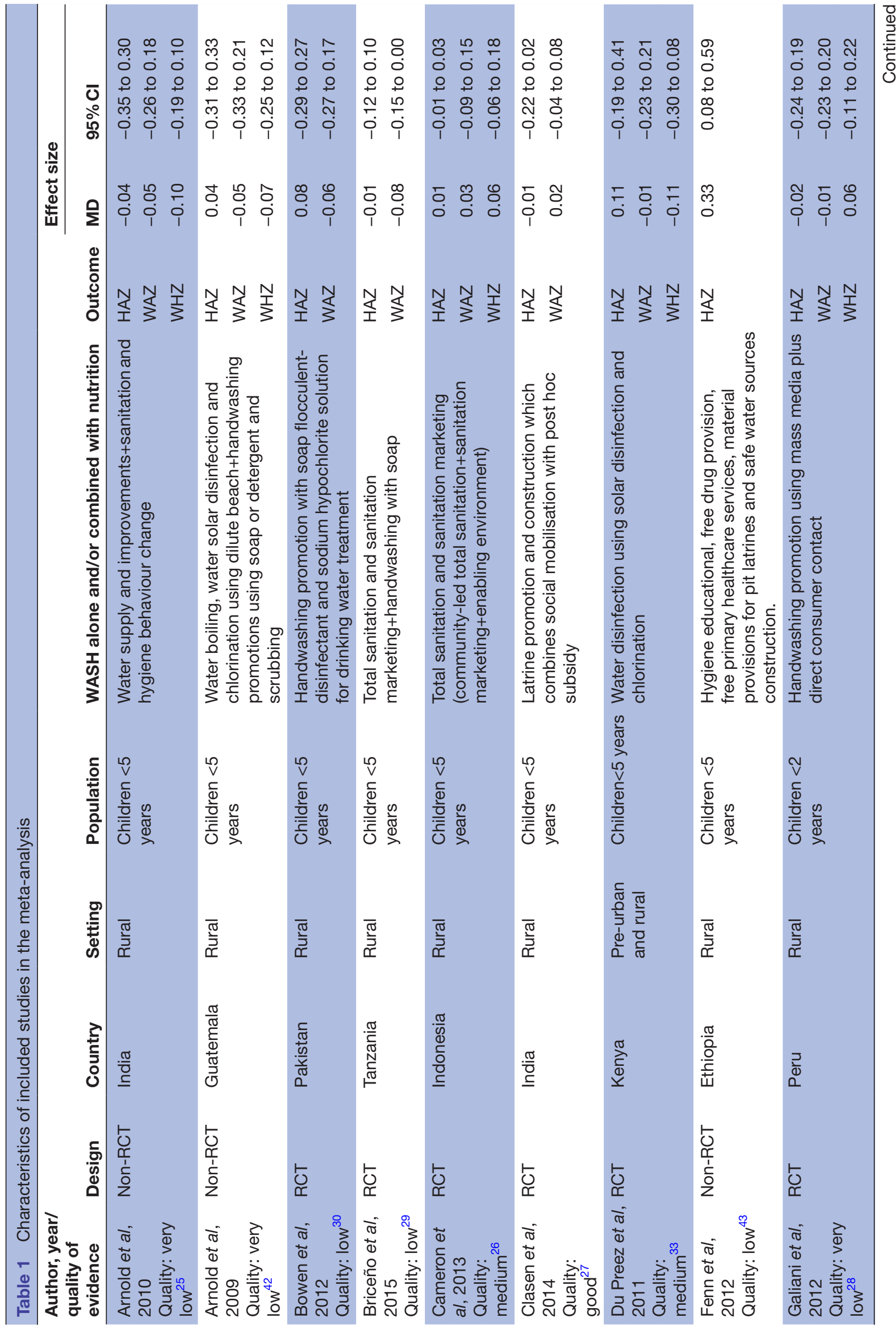

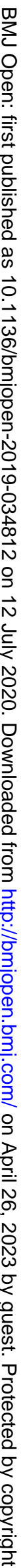




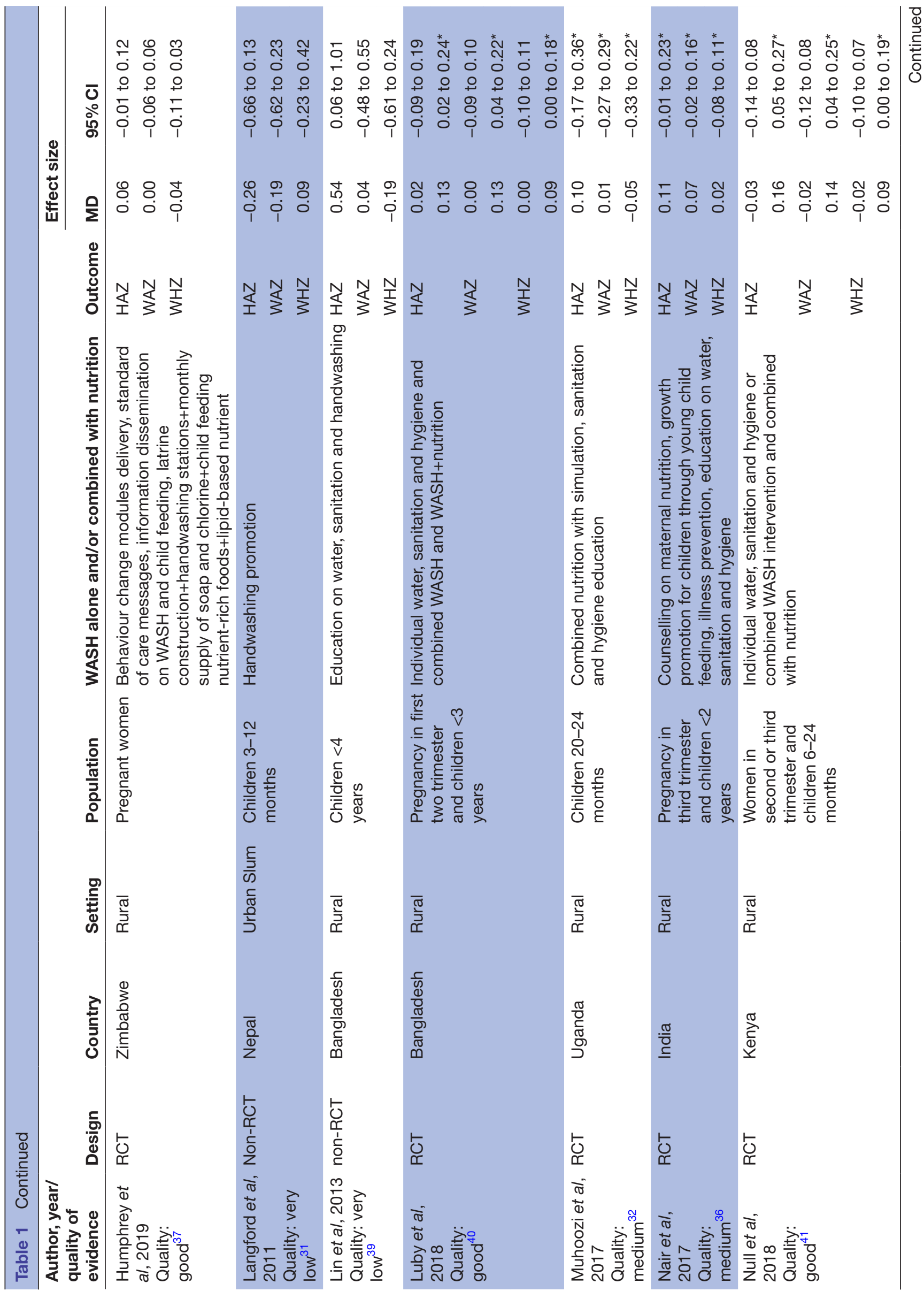

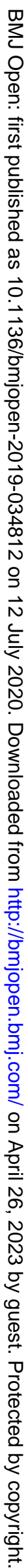




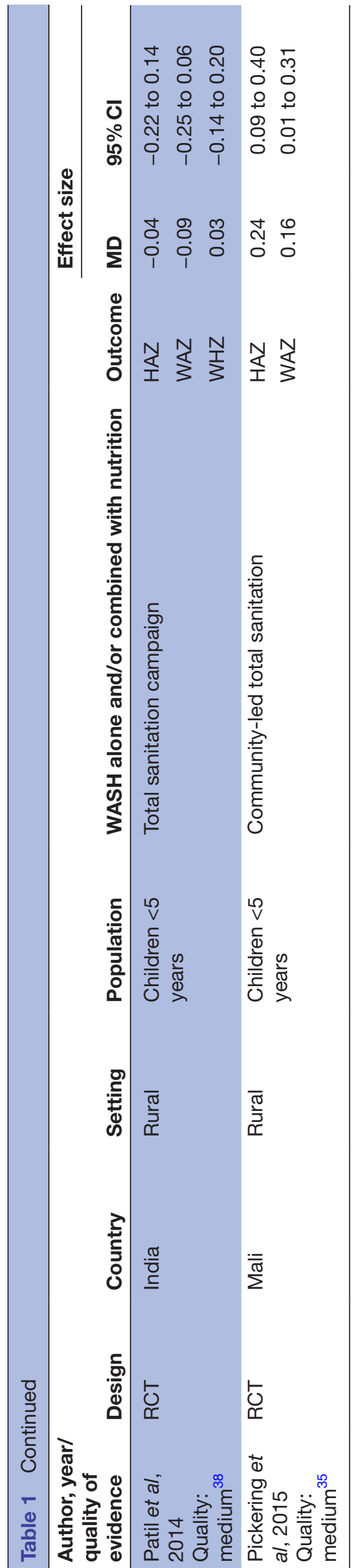

statistically significant effect on HAZ $(\mathrm{MD}=0.03$; $95 \% \mathrm{CI}$ -0.01 to 0.06 ) (figure 2 ).

Subgroup analysis of children aged $\leq 2$ years in the intervention group showed an increase in $\mathrm{HAZ}(\mathrm{MD}=0.07$; $95 \%$ CI 0.01 to $0.13, \mathrm{p}=0.02$ ) but not for children aged $<5$ years $(\mathrm{MD}=0.05 ; 95 \% \mathrm{CI}-0.00$ to 0.11 ) (table 2 ) and (online supplementary figure 1). WASH interventions alone, that were delivered over 18-60 months, showed a positive effect on children's HAZ (MD $=0.04$; 95\% CI 0.01 to $0.08, \mathrm{p}=0.02)$. Non-RCTs indicated a stronger effect ( $\mathrm{MD}=0.15 ; 95 \% \mathrm{CI} 0.07$ to $0.23, \mathrm{p}=0.0003$ ) compared with RCTs (MD $=0.02 ; 95 \%$ CI -0.02 to 0.07$)$. WASH interventions alone in sub-Saharan Africa has increased HAZ $(\mathrm{MD}=0.06 ; 95 \%$ CI 0.01 to $0.10, \mathrm{p}=0.01)$.

\section{Individual WASH components effect on HAZ}

We also examined the effect of individual WASH components on HAZ for water, sanitation and handwashing separately. None of the individual interventions showed improvement in HAZ: water only interventions (MD=-0.06; $95 \% \mathrm{CI}:-0.13$ to 0.01$)$; sanitation only interventions ( $\mathrm{MD}=-0.01 ; 95 \% \mathrm{CI}$ : -0.10 to 0.08$)$; and handwashing only interventions ( $\mathrm{MD}=-0.06 ; 95 \% \mathrm{CI}:-0.16$ to 0.04) (plots not shown).

\section{Combined WASH components effect on HAZ}

We examined the effect of integrated WASH using studies that included at least two components (i.e., water, sanitation and handwashing). The pooled estimate from nonRCT studies showed an effect on HAZ (MD $=0.15$; $95 \%$ CI 0.07 to 0.23 , $\mathrm{p}=0.0003)$ compared with $\mathrm{RCTs}(\mathrm{MD}=-0.02$; $95 \% \mathrm{CI}-0.07$ to 0.03$)$. The overall pooled estimate from non-RCTs and RCTs together, with substantial heterogeneity $\left(I^{2}=76 \%\right)$, showed no effect on HAZ (MD $=0.02$; $95 \%$ CI -0.02 to 0.07 ) (figure 3 ).

\section{Combined WASH with nutrition interventions effect on HAZ}

Five RCT studies reported combined WASH with nutrition versus control. ${ }^{32} 36374041$ We compared intervention groups which received WASH plus nutrition versus nutrition (MD $=-0.01 ; 95 \% \mathrm{CI}-0.13$ to 0.12 ) and WASH plus nutrition versus WASH $(\mathrm{MD}=18 ; 95 \% \mathrm{CI} 0.12$ to $0.25, \mathrm{p}<0.001)$. Strong evidence of the effect on HAZ was indicated in groups that received WASH plus nutrition versus no-WASH plus no-nutrition ( $\mathrm{MD}=0.13 ; 95 \% \mathrm{CI}$ 0.08 to $0.17, \mathrm{p}<0.001$ ), with no evidence of heterogeneity $\left(I^{2}=0 \%\right)$. One study ${ }^{32}$ found no effect of combined WASH with nutrition interventions versus control on HAZ ( $\mathrm{MD}=0.10 ; 95 \% \mathrm{CI}-0.08$ to 0.28 ) (figure 4 ).

\section{Weight-for-age}

Regardless of WASH components included in the study, data on WAZ were reported in 4 non-RCTs and 11 RCTs which were included in the meta-analysis. Of the nonRCTs, only one study ${ }^{39}$ showed a significant effect on WAZ $(\mathrm{MD}=0.42 ; 95 \% \mathrm{CI} 0.02$ to 0.82$)$ and the pooled estimate was non-significant ( $\mathrm{MD}=-0.01 ; 95 \% \mathrm{CI}-0.16$ to 0.14$)$, with moderate heterogeneity $\left(I^{2}=46 \%\right)$. Only one ${ }^{35}$ of the 11 RCTs showed a positive effect on WAZ (MD $=0.09$; 


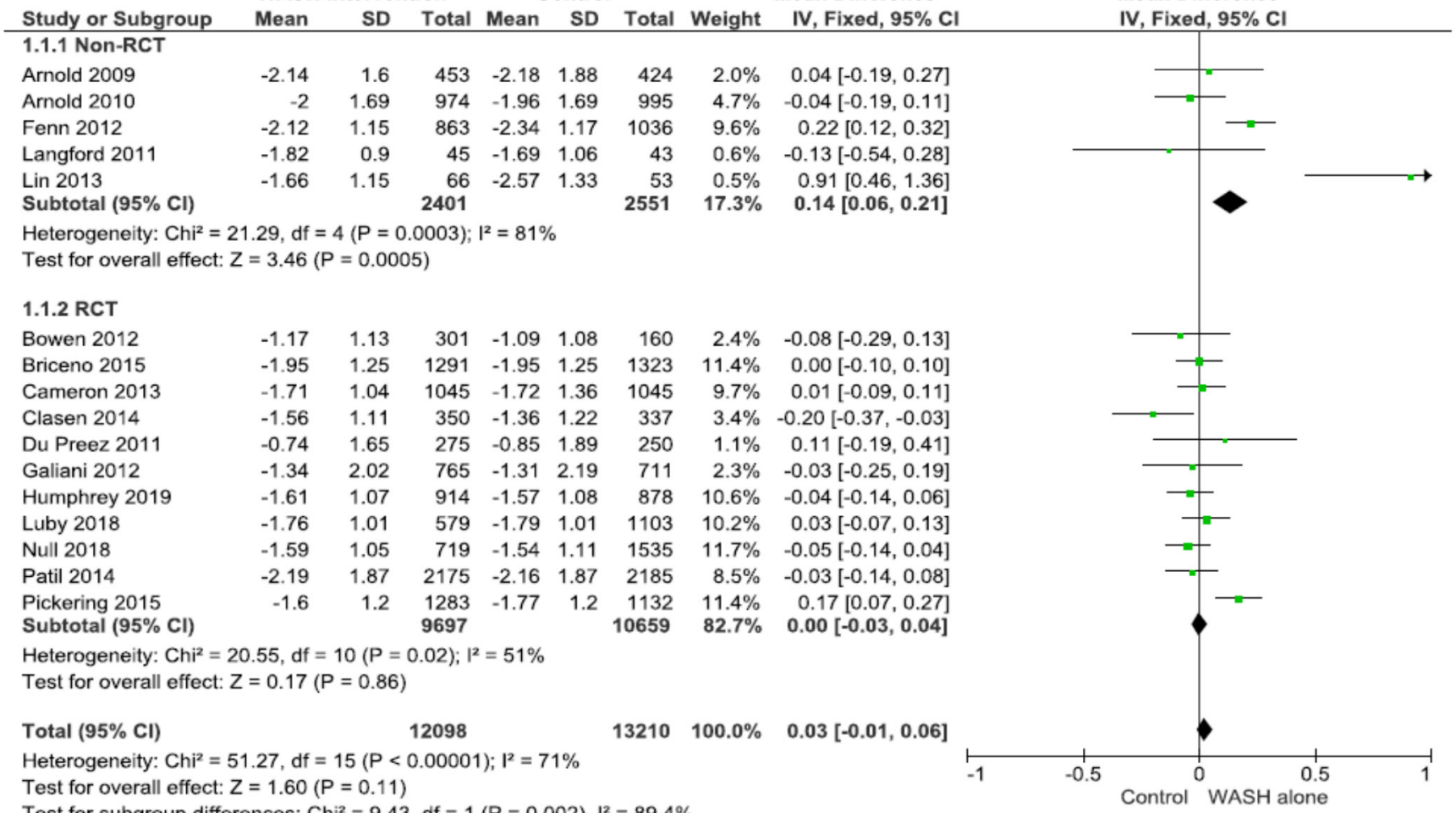

Test for subgroup differences: $\mathrm{Ch}^{2}=9.43, \mathrm{df}=1(\mathrm{P}=0.002), \mathrm{I}^{2}=89.4 \%$

95\% CI 0.01 to 0.17 ) and the pooled estimate of nonRCTs and RCTs together was non-significant $(\mathrm{MD}=-0.02$; $95 \%$ CI -0.05 to 0.00$)$ with low heterogeneity $\left(I^{2}=30 \%\right)$ (online supplementary figure 2). Subgroup analysis of the overall pooled estimate did not show evidence of an effect on WAZ for children $<2$ years of age, but one study $^{35}$ showed evidence of an effect $(\mathrm{MD}=0.15 ; 95 \% \mathrm{CI}$ 0.04 to 0.26$)$ compared with children $<5$ years $(\mathrm{MD}=0.09$; $95 \% \mathrm{CI} 0.01$ to 0.17$)$. WASH interventions alone showed a negative effect on WAZ in the Southern Asia region $(\mathrm{MD}=-0.04 ; 95 \% \mathrm{CI}-0.10$ to -0.01$)$ (table 2$)$.

\section{Combined WASH with nutrition interventions effect on WAZ}

We included five RCT studies which reported combined WASH with nutrition versus control. ${ }^{32} 36374041$ Combined WASH with nutrition interventions improved WAZ ( $\mathrm{MD}=0.09 ; 95 \%$ CI 0.05 to $0.13, \mathrm{p}<0.001$ ) with minimal heterogeneity $\left(I^{2}=2 \%\right)$. However, two studies ${ }^{32} 36$ did not show a positive effect (figure 5). We found only three WASH studies ${ }^{3740} 41$ which reported WASH plus nutrition versus nutrition and WASH plus nutrition versus WASH on WAZ. Using these three studies, we found $\mathrm{MD}=0.00$; $95 \% \mathrm{CI}-0.11$ to 0.12 for WASH plus nutrition versus nutrition and $\mathrm{MD}=0.16$; $95 \%$ CI 0.11 to 0.22 for WASH plus nutrition versus WASH interventions.

\section{Weight-for-height}

Data on WHZ were available in four non-RCTs and seven RCTs, and no evidence of an effect was detected from both non-RCTs (MD $=-0.10 ; 95 \% \mathrm{CI}-0.19$ to 0.00$)$ and RCTs ( $\mathrm{MD}=-0.01 ; 95 \% \mathrm{CI}-0.05$ to 0.03 ) (online supplementary figure 3). There was no evidence of heterogeneity in both non-RCTs and RCTs $\left(I^{2}=0 \%\right)$. The pooled estimate of non-RCT studies and RCTs together did not show a statistically significant effect on WHZ (MD $=-0.03 ; 95 \%$ CI -0.06 to 0.01$)$. Although the heterogeneity was minimal $\left(I^{2}=3 \%\right)$, a borderline effect was detected for WHZ from combined WASH with nutrition interventions $(\mathrm{MD}=0.04$; 95\% CI 0.00 to $0.09, \mathrm{p}=0.04$ ) (plot not shown). Using three studies we found $\mathrm{MD}=0.01 ; 95 \% \mathrm{CI}-0.07$ to 0.09 for WASH plus nutrition versus nutrition and $\mathrm{MD}=0.10$; $95 \%$ CI 0.05 to 0.16 for WASH plus nutrition versus WASH interventions.

\section{Publication bias and sensitivity analysis}

The funnel plot shows there was no evidence of publication bias for all studies. The plot was symmetrical (online supplementary figure 4); Egger's test provided a $p$ value of 0.976 for bias. There was also no evidence of publication bias detected from separate plots for RCTs and nonRCTs (online supplementary figure 5); Egger's test gave $p$ values of 0.873 for non-RCT and 0.365 for RCTs, respectively. Omitting each study did not influence the pooled estimate, and results from fixed and random effects models were not different (data not shown).

\section{Meta-regression}

The coefficients of regressed effect measures on the moderators (general domain, risk of bias and items that measure risk of bias) are given in table 3. The effect measure was increased with baseline outcome measurements between intervention and control groups $(\mathrm{p}=0.007)$ 


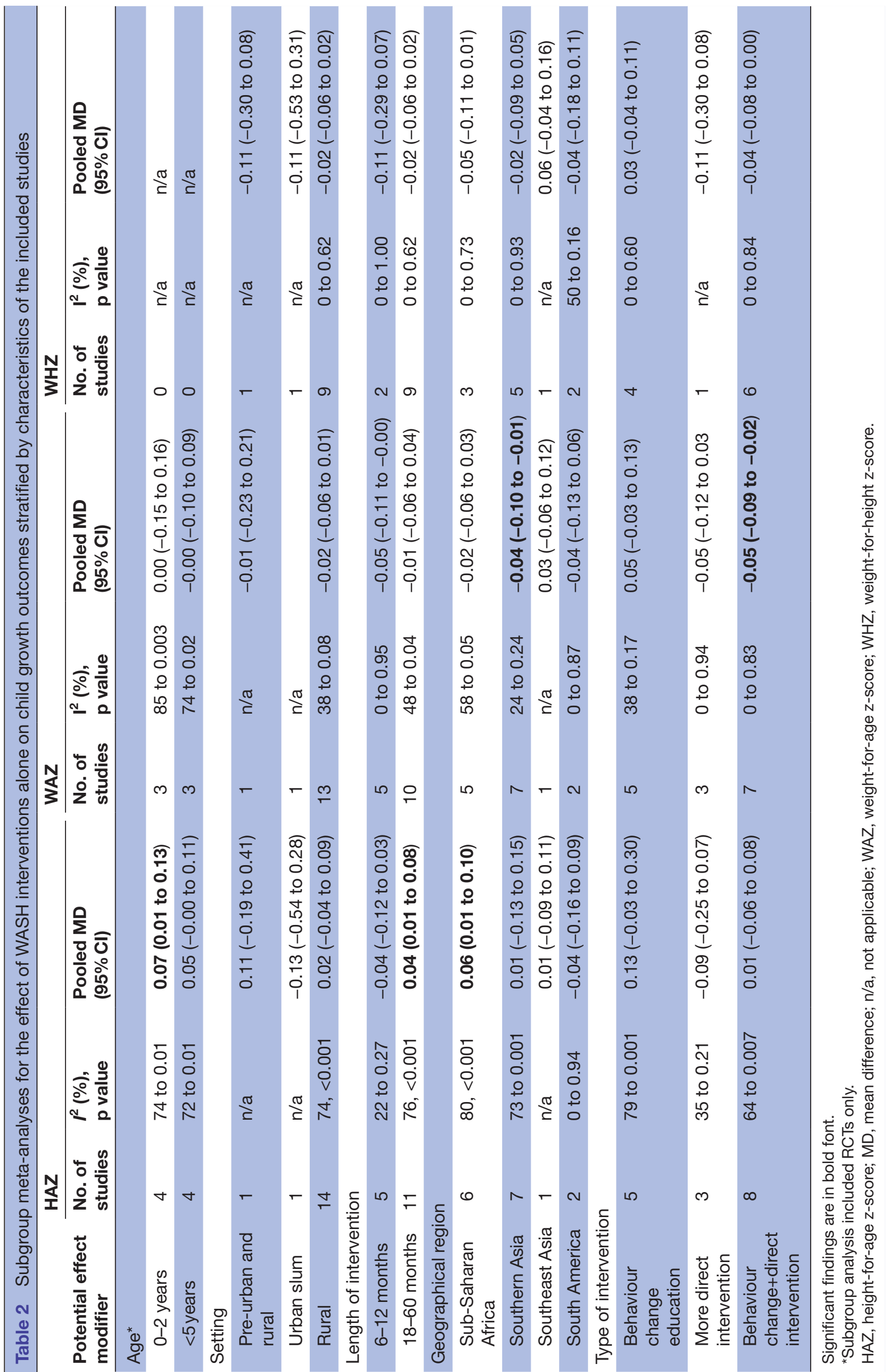




\begin{tabular}{|c|c|c|c|c|c|c|c|c|c|c|c|c|}
\hline \multirow{2}{*}{$\begin{array}{l}\text { Study or Subgroup } \\
1.21 .1 \text { Non-RCTs }\end{array}$} & \multicolumn{3}{|c|}{ WASH intervention } & \multicolumn{3}{|c|}{ Control } & \multicolumn{3}{|c|}{ Mean Difference } & \multirow{2}{*}{$\begin{array}{l}\text { Mean Difference } \\
\text { IV, Fixed, } 95 \% \mathrm{Cl}\end{array}$} & & \\
\hline & Mean & SD & Total & Mean & SD & Total & Weight & IV, Fixed, $95 \% \mathrm{Cl}$ & & & & \\
\hline Arnold 2009 & -2.14 & 1.6 & 453 & -2.18 & 1.88 & 424 & $3.1 \%$ & $0.04[-0.19,0.27]$ & & & & \\
\hline Fenn 2012 & -2.12 & 1.15 & 863 & -2.34 & 1.17 & 1036 & $15.2 \%$ & $0.22[0.12,0.32]$ & & . & & \\
\hline $\begin{array}{l}\text { Lin } 2013 \\
\text { Subtotal }(95 \% \mathrm{Cl})\end{array}$ & -1.66 & 1.15 & $\begin{array}{r}66 \\
2356\end{array}$ & -2.57 & 1.33 & $\begin{array}{r}53 \\
2508\end{array}$ & $\begin{array}{r}0.8 \% \\
26.6 \%\end{array}$ & $\begin{array}{l}0.91[0.46,1.36] \\
0.15[0.07,0.23]\end{array}$ & & & & \\
\hline \multicolumn{13}{|l|}{ 1.21.2 RCTs } \\
\hline Bowen 2012 & -1.17 & 1.13 & 301 & -1.09 & 1.08 & 160 & $3.8 \%$ & $-0.08[-0.29,0.13]$ & & & & \\
\hline Briceno 2015 & -1.95 & 1.25 & 1291 & -1.95 & 1.25 & 1323 & $18.1 \%$ & $0.00[-0.10,0.10]$ & & & & \\
\hline Humphrey 2019 & -1.61 & 1.07 & 914 & -1.57 & 1.08 & 878 & $16.8 \%$ & $-0.04[-0.14,0.06]$ & & 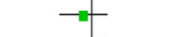 & & \\
\hline Luby 2018 & -1.76 & 1.01 & 579 & -1.79 & 1.01 & 1103 & $16.1 \%$ & $0.03[-0.07,0.13]$ & & & & \\
\hline \multicolumn{13}{|c|}{ Heterogeneity: $\mathrm{Ch}^{2}=1.95, \mathrm{df}=4(\mathrm{P}=0.75) ; \mathrm{I}^{2}=0 \%$} \\
\hline Total $(95 \% \mathrm{Cl})$ & & & 6160 & & & 7507 & $100.0 \%$ & $0.02[-0.02,0.07]$ & & & & \\
\hline $\begin{array}{l}\text { Heterogeneity: } \mathrm{Chi}^{2}= \\
\text { Test for overall effect } \\
\text { Test for subgroup diff }\end{array}$ & $\begin{array}{l}.00, \mathrm{df}= \\
=1.20( \\
\text { ences: } \mathrm{C}\end{array}$ & $\begin{array}{l}B(P<C \\
=0.23 \\
{ }^{2}=12 .\end{array}$ & $.0001)$ & $\begin{array}{l}\left.\right|^{2}=76 \% \\
1(P=0\end{array}$ & $\%$ & ${ }^{2}=$ & & & -0.5 & Control WASH & 0.5 & 1 \\
\hline
\end{tabular}

\section{DISCUSSION}

We reviewed evidence of the effect of WASH interventions alone, separately, and when combined with nutrition on child growth. Our review focused on three child growth measures: HAZ, WAZ and WHZ. In this review, WASH interventions alone from non-RCTs were found to improve HAZ compared with RCTs. The overall pooled estimate from both non-RCTs and RCTs together indicated no effect of WASH interventions alone on HAZ. The findings of the current study do not support the previous research by Gera $e t$ al which showed that WASH interventions improved HAZ by $22 \% .{ }^{12}$ The meta-analysis from five RCTs conducted by Dangour and colleagues showed a borderline effect on HAZ. ${ }^{11}$ Another meta-analysis by Freeman et al showed a borderline effect of WASH interventions after pooling non-RCTs and RCTs together. ${ }^{17}$ RCT studies failed to show evidence of an effect on HAZ. A possible explanation for this might be that population level WASH interventions using a community approach may have an increased positive effect compared with household level interventions. In addition, long-term exposure to high quality hygiene and sanitation may be required to improve child linear growth. There is a lesson to be learned from three RCTs that showed WASH interventions improved diarrhoea in Bangladesh ${ }^{40}$ but not in Kenya ${ }^{41}$ and Zimbabwe. ${ }^{37}$ However, one plausible explanation is that there may have been unmeasured confounding variables in the non-RCT studies.

Regardless of the duration of interventions and age range of study subjects, the value of the effect estimate in the current study suggests a weak link may exist between WASH interventions alone and HAZ. There are several plausible reasons for weak evidence of an effect. First, some studies in the current review were studies of WASH intervention programme evaluations. These evaluations assessed the capability of specific interventions under low uptake, low adherence and with a high risk of contamination that may have contributed to underestimating the true effect of WASH interventions. Second, the analysis of reviews was based on a combination of individual and/or combined WASH components which may have contributed

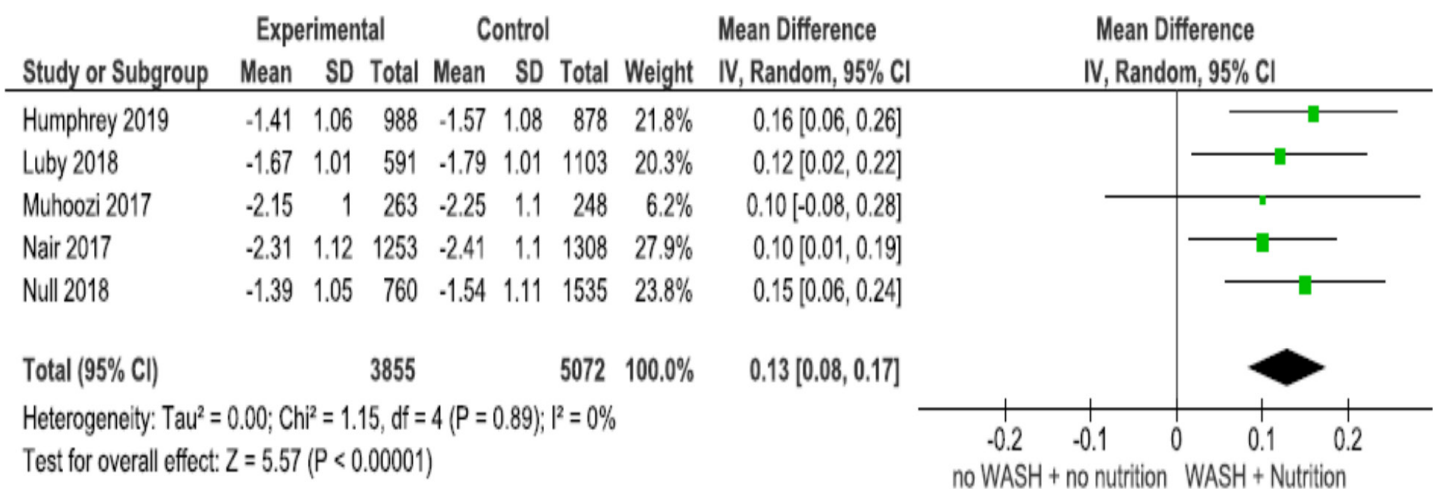

Figure 4 Forest plot of water, sanitation and hygiene (WASH)+nutrition versus no-WASH+no-nutrition for height-for-age zscore. 


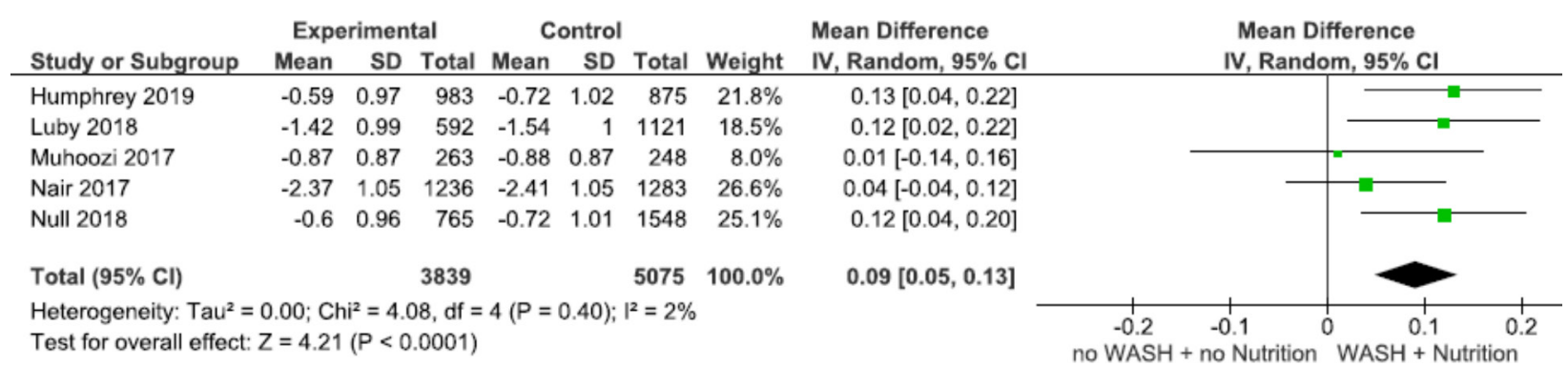

Figure 5 Forest plot of water, sanitation and hygiene (WASH)+nutrition versus no-WASH+no nutrition for weight-for-age zscore.

to discrepancies in effect size. The current review found a positive effect of WASH interventions from non-RCTs on linear growth when at least two components of WASH were provided together. These findings further support the idea of previous studies ${ }^{44} 45$ that integrated components of WASH may be more effective compared with single interventions. The age of the child receiving the interventions is also important, as shown in subgroup analyses.

Children $<2$ years of age were more likely to respond to WASH interventions compared with children $<5$ years for linear growth. This result matches that observed in a previous review. ${ }^{11}$ The current review supports previous studies which suggested that interventions should focus on the first 1000 days of a child's life. ${ }^{46-48}$ The first 1000 days of life are characterised by rapid growth, high nutrition requirements, greater susceptibility to infections, and full dependence on others for care, nutrition and high social interaction. ${ }^{46}$ Duration of the interventions also appears to be important in the current study. WASH interventions that were delivered over a duration of 18-60 months showed improved HAZ compared with interventions delivered over $6-12$ months. This result is a new and novel finding that has not been described in previous reviews. One explanation is that WASH is often interlaced with other complex interventions that require behavioural change over an extended period to see any positive effect. Also, linear growth failure typically arises from chronic undernutrition which requires long-term adherence to interventions to observe positive change. ${ }^{49}$

This review also found, for the first time, a significant positive effect of combined WASH with nutrition interventions on child's HAZ. The observed improvement in linear growth could be attributed to the synergistic effect of combined WASH with nutrition interventions. This finding has an important implication for developing an integrated intervention approach for WASH and nutrition sectors in order to tackle child linear growth failure. A previous study suggested $90 \%$ coverage of nutrition interventions would only decrease stunting by $20 \%,{ }^{13}$ while interventions such as WASH shares a large portion of the remaining burden. ${ }^{9}$ There were lessons we can learn from the WASH-Benefit studies $^{40}{ }^{41}$ and Sanitation Hygiene Infant Nutrition Efficacy (SHINE) trail ${ }^{37}$ in which child feeding interventions slightly (12\% points) increased child linear growth. Overall,

Table 3 Results of meta-regression analyses of mean difference in child growth on quality scores items of the included studies

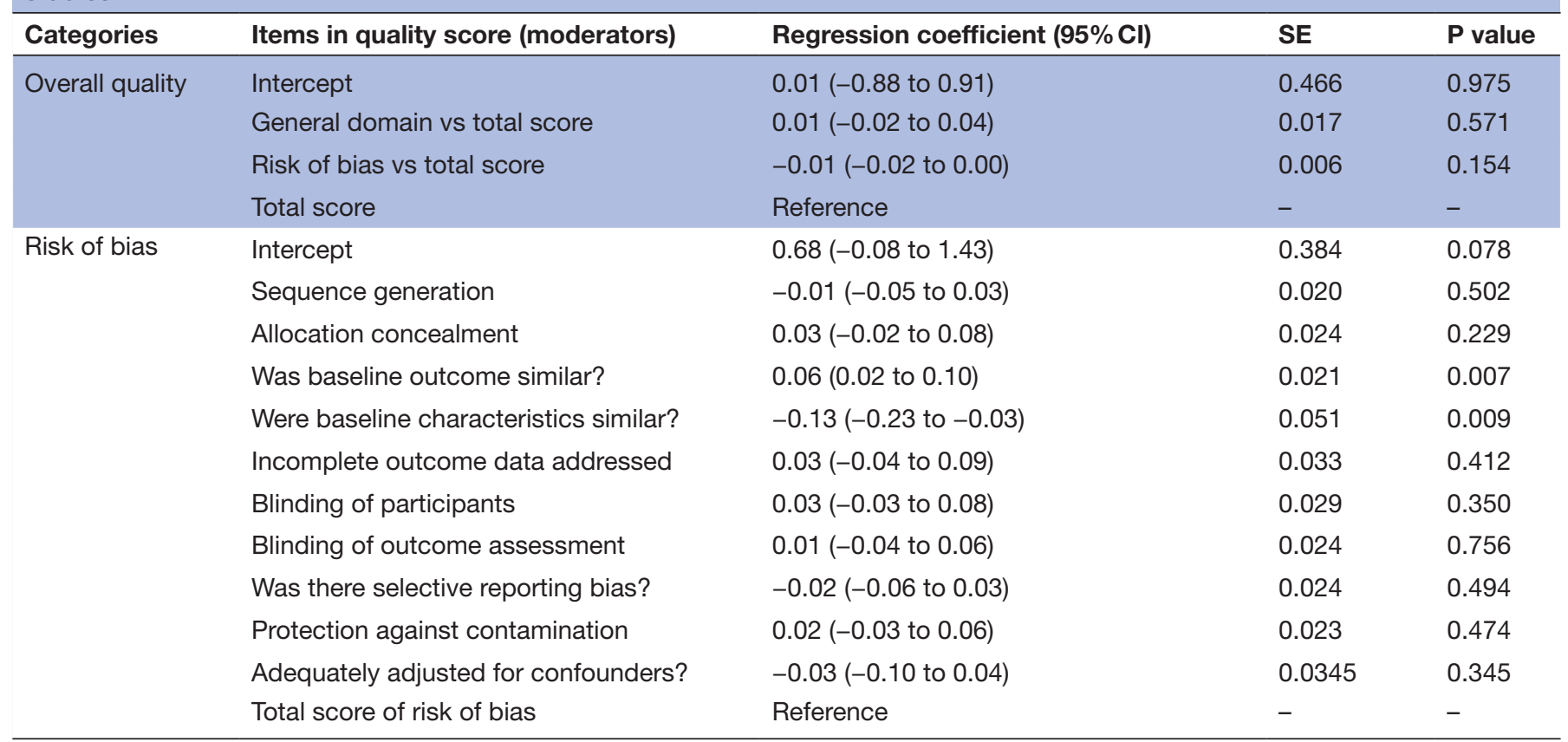


there appears to be evidence to indicate that the component of intervention, either WASH alone or combined with nutrition, age of child, and duration of interventions, have an impact on child growth.

The current review did not detect evidence of benefit from WASH interventions alone for weight growth (WAZ). The present findings seem to be consistent with other research which found no evidence of an effect from WASH interventions alone. ${ }^{11}$ However, the findings of the current review do not support previous reviews which found WASH interventions alone improved WAZ of children. ${ }^{12}{ }^{17}$ The current study results must be interpreted with caution because the Gera $e t a l$ study found a small positive effect on WAZ from water supply and water treatment interventions only, whereas the Freeman et al review was based on sanitation only. The results of the current review from subgroup analysis by age and exposure duration to interventions did not show a significant benefit from WASH interventions alone for WAZ. However, the combined interventions of WASH with nutrition was found to improve WAZ. In reviewing the literature, there appears to be no published evidence on the effect of combined WASH with nutrition interventions on WAZ, making this a new finding in the research literature. Being a new finding, further studies and reviews will be important to undertake to add further support to these findings. In the current review, we found no evidence of an effect on WHZ from WASH interventions alone and a borderline effect from combined WASH interventions with nutrition. The findings are consistent with previous reviews that detected no evidence of an effect of WASH interventions on WHZ. ${ }^{12} 17$ Subgroup analysis based on age and interventions duration also did not change the value of WHZ in the current review.

\section{Strengths and limitations}

This study reviewed evidence from studies that were conducted in low and middle-income countries following the guidelines of PRISMA. We explored data from both RCTs and non-randomised studies. Our analyses are grouped into key indicators which show a clear summary of intervention outcomes. Sources of heterogeneity were assessed using subgroup analysis and meta-regressions based on the known factors. Most of the methodological quality of the included studies was deemed very low, low and medium, that is, none of the included studies had low risk of bias. We included few studies in the meta-analysis of individual WASH components alone and combined with nutrition due to the lack of consistency in reporting outcomes and summary statistics. Therefore, true heterogeneity between studies and correct publication bias may not have been detected. As few studies were included in the meta-analysis, this study could not confirm that the effect of WASH plus nutrition versus nutrition or WASH on child growth may be due to nutrition only.

\section{CONCLUSIONS}

The present review found that HAZ was more responsive to WASH interventions alone that were delivered over
18-60 months and among children $<2$ years of age. HAZ could be improved through combined WASH with nutrition interventions. Improvement in WAZ was indicated in a group with combined WASH with nutrition interventions. Although no evidence of an effect was found from WASH interventions alone on WHZ, we found a borderline effect when combined with nutrition. Well-designed epidemiological studies are recommended that examine the minimum length of intervention necessary to be effective and the role of behavioural aspects including social norms in the implementation of WASH interventions. WASH and nutrition interventions should be considered together when designing strategies to tackle CGF with a particular focus on the first 1000 days of a child's life.

Acknowledgements The authors are grateful to The University of New South Wales (UNSW), Australia, for providing a full scholarship to the primary author. Our acknowledgement goes to the School of Public Health and Community Medicine, UNSW, for providing work resources for the primary author. We also extend our thanks to the UNSW Library research consultants for their support in searching material for this systematic review.

Contributors TB conceived the idea, searched and screened all relevant articles, extracted the data, performed analysis, assessed quality and drafted the manuscript. PR and BR checked the screened and excluded studies by the primary author and edited the manuscript.

Funding This study was conducted as part of a PhD thesis. The $\mathrm{PhD}$ is funded by the Graduate Research Scholarship of the Australian Government and the UNSW, as well as the School of Public Health and Community Medicine.

Competing interests None declared.

Patient and public involvement Patients and/or the public were not involved in the design, or conduct, or reporting or dissemination plans of this research.

Patient consent for publication Not required.

Provenance and peer review Not commissioned; externally peer reviewed.

Data availability statement All data relevant to the study are provided in the article or given as supplementary file. All data used in this study are from an open access literature.

Open access This is an open access article distributed in accordance with the Creative Commons Attribution Non Commercial (CC BY-NC 4.0) license, which permits others to distribute, remix, adapt, build upon this work non-commercially, and license their derivative works on different terms, provided the original work is properly cited, appropriate credit is given, any changes made indicated, and the use is non-commercial. See: http://creativecommons.org/licenses/by-nc/4.0/.

ORCID iD

Tolesa Bekele http://orcid.org/0000-0002-9335-0012

\section{REFERENCES}

1 Wang $Y$, Hsin-Jen C. Use of percentiles and Z -scores in anthropometry. In: Handbook of anthropometry: physical measures of human form in health and disease. New York: Springer, 2012: 29-48.

2 Aguayo VM, Menon P. Stop stunting: improving child feeding, women's nutrition and household sanitation in South Asia. Matern Child Nutr 2016;12(Suppl 1):3-11.

3 Arnold BF, Null C, Luby SP, et al. Cluster-randomised controlled trials of individual and combined water, sanitation, hygiene and nutritional interventions in rural Bangladesh and Kenya: the WASH benefits study design and rationale. BMJ Open 2013;3:e003476.

4 USAID, UNICEF, WHO. Improving nutrition outcomes with better water, sanitation and hygiene: practical solutions for policies and programmes. Geneva: World Health Organization, 2015. https://apps. who.int/iris/handle/10665/193991

5 Bain R, Cronk R, Hossain R, et al. Global assessment of exposure to faecal contamination through drinking water based on a systematic review. Trop Med Int Health 2014;19:917-27.

6 Cha S, Kang D, Tuffuor B, et al. The effect of improved water supply on diarrhea prevalence of children under five in the Volta region of 
Ghana: a cluster-randomized controlled trial. Int J Environ Res Public Health 2015:12:12127-43.

7 Freeman MC, Stocks ME, Cumming O, et al. Hygiene and health: systematic review of handwashing practices worldwide and update of health effects. Trop Med Int Health 2014;19:906-16.

8 Ngure FM, Reid BM, Humphrey JH, et al. Water, sanitation, and hygiene (WASH), environmental enteropathy, nutrition, and early child development: making the links. Ann N Y Acad Sci 2014;1308:118-28.

9 Chase C, Ngure FM. Multisectoral approaches to improving nutrition: water, sanitation, and hygiene. Water and sanitation program. Washington DC: World Bank, 2016. http://documents.worldbank. org/curated/en/881101468196156182/Multisectoral-approaches-toimproving-nutrition-water-sanitation-and-hygiene

10 Belizario VY, Liwanag HJC, Naig JRA, et al. Parasitological and nutritional status of school-age and preschool-age children in four villages in southern Leyte, Philippines: lessons for monitoring the outcome of Community-Led total sanitation. Acta Trop 2015:141:16-24.

11 Dangour AD, Watson L, Cumming O, et al. Interventions to improve water quality and supply, sanitation and hygiene practices, and their effects on the nutritional status of children. Cochrane Database Syst Rev 2013:CD009382.

12 Gera T, Shah D, Sachdev HS. Impact of water, sanitation and hygiene interventions on growth, Non-diarrheal morbidity and mortality in children residing in low- and middle-income countries: a systematic review. Indian Pediatr 2018;55:381-93.

13 Bhutta ZA, Das JK, Rizvi A, et al. Evidence-based interventions for improvement of maternal and child nutrition: what can be done and at what cost? Lancet 2013;382:452-77.

14 Humphrey $\mathrm{JH}$. Child undernutrition, tropical enteropathy, toilets, and handwashing. Lancet 2009;374:1032-5.

15 Ashorn P, Alho L, Ashorn U, et al. Supplementation of maternal diets during pregnancy and for 6 months postpartum and infant diets Thereafter with Small-Quantity lipid-based nutrient supplements does not promote child growth by 18 months of age in rural Malawi: a randomized controlled trial. J Nutr 2015;145:1345-53.

16 Krebs NF, Mazariegos M, Chomba E, et al. Randomized controlled trial of meat compared with multimicronutrient-fortified cereal in infants and toddlers with high stunting rates in diverse settings. Am J Clin Nutr 2012;96:840-7.

17 Freeman MC, Garn JV, Sclar GD, et al. The impact of sanitation on infectious disease and nutritional status: a systematic review and meta-analysis. Int J Hyg Environ Health 2017;220:928-49.

18 Moher D, Liberati A, Tetzlaff J, et al. Preferred reporting items for systematic reviews and meta-analyses: the PRISMA statement. PLOS Med 2009;6:e100009.

19 Onis MD, Blössner M. WHO global database on child growth and malnutrition. Geneva: World Health Organization, 1997.

20 EPOC. Cochrane effective practice and organisation of care (EPOC). suggested risk of bias criteria for EPOC reviews, 2017. Available: http://epoc.cochrane.org/resources/epoc-resources-review-authors [Accessed 15 May 2018].

21 Chalmers TC, Smith H, Blackburn B, et al. A method for assessing the quality of a randomized control trial. Control Clin Trials 1981:2:31-49.

22 Jadad AR, Moore RA, Carroll D, et al. Assessing the quality of reports of randomized clinical trials: is blinding necessary? Control Clin Trials 1996;17:1-12.

23 The Cochrane Collaboration. Review Manager (RevMan) [Computer program] [program]. 5.3 version. Copenhagen: The Nordic Cochrane Centre, 2014.

24 Higgins JPT, Thompson SG. Quantifying heterogeneity in a metaanalysis. Stat Med 2002;21:1539-58.

25 Arnold BF, Khush RS, Ramaswamy P, et al. Causal inference methods to study nonrandomized, preexisting development interventions. Proc Natl Acad Sci U S A 2010;107:22605-10.

26 Cameron L, Shah M, Susan O. Impact evaluation of a large-scale rural sanitation project in Indonesia. Policy research working paper 6360: series number 83. World Bank, 2013.

27 Clasen T, Boisson S, Routray P, et al. Effectiveness of a rural sanitation programme on diarrhoea, soil-transmitted helminth infection, and child malnutrition in Odisha, India: a clusterrandomised trial. Lancet Glob Health 2014;2:e645-53.

28 Galiani S, Gertler P, Alexandra OV. The effect of large-scale MassMedia and community level interventions. promoting handwashing behavior in Peru. Policy research working paper 6257: series number 74. World Bank, 2012.

29 Briceño B, Coville A, Martinez S. Promoting handwashing and sanitation evidence from a large-scale randomized trial in rural Tanzania. Policy research working paper 7164. World Bank, 2015.

30 Bowen A, Agboatwalla M, Luby S, et al. Association between intensive handwashing promotion and child development in Karachi, Pakistan: a cluster randomized controlled trial. Arch Pediatr Adolesc Med 2012;166:1037-44.

31 Langford R, Lunn P, Panter-Brick C. Hand-washing, subclinical infections, and growth: a longitudinal evaluation of an intervention in Nepali slums. Am J Hum Biol 2011;23:621-9.

32 Muhoozi GKM, Atukunda P, Diep LM, et al. Nutrition, hygiene, and stimulation education to improve growth, cognitive, language, and motor development among infants in Uganda: a cluster-randomized trial. Matern Child Nutr 2018;14:e12527.

33 du Preez M, Conroy RM, Ligondo S, et al. Randomized intervention study of solar disinfection of drinking water in the prevention of dysentery in Kenyan children aged under 5 years. Environ Sci Technol 2011:45:9315-23.

34 Boisson S, Stevenson M, Shapiro L, et al. Effect of household-based drinking water chlorination on diarrhoea among children under five in Orissa, India: a double-blind randomised placebo-controlled trial. PLoS Med 2013;10:e1001497.

35 Pickering AJ, Djebbari H, Lopez C, et al. Effect of a communityled sanitation intervention on child diarrhoea and child growth in rural Mali: a cluster-randomised controlled trial. Lancet Glob Health 2015;3:e701-11.

36 Nair N, Tripathy P, Sachdev HS, et al. Effect of participatory women's groups and counselling through home visits on children's linear growth in rural eastern India (CARING trial): a cluster-randomised controlled trial. Lancet Glob Health 2017;5:e1004-16.

37 Humphrey JH, Mbuya MNN, Ntozini R, et al. Independent and combined effects of improved water, sanitation, and hygiene, and improved complementary feeding, on child stunting and anaemia in rural Zimbabwe: a cluster-randomised trial. Lancet Glob Health 2019; 7:e132-47.

38 Patil SR, Arnold BF, Salvatore AL, et al. The effect of India's total sanitation campaign on defecation behaviors and child health in rural Madhya Pradesh: a cluster randomized controlled trial. PLoS Med 2014;11:e1001709.

39 Lin A, Arnold BF, Afreen S, et al. Household environmental conditions are associated with enteropathy and impaired growth in rural Bangladesh. Am J Trop Med Hyg 2013;89:130-7.

40 Luby SP, Rahman M, Arnold BF, et al. Effects of water quality, sanitation, handwashing, and nutritional interventions on diarrhoea and child growth in rural Bangladesh: a cluster randomised controlled trial. Lancet Glob Health 2018;6:e302-15.

41 Null C, Stewart CP, Pickering AJ, et al. Effects of water quality, sanitation, handwashing, and nutritional interventions on diarrhoea and child growth in rural Kenya: a cluster-randomised controlled trial. Lancet Glob Health 2018;6:e316-29.

42 Arnold B, Arana B, Mäusezahl D, et al. Evaluation of a pre-existing, 3 -year household water treatment and handwashing intervention in rural Guatemala. Int J Epidemiol 2009;38:1651-61.

43 Fenn B, Bulti AT, Nduna T, et al. An evaluation of an operations research project to reduce childhood stunting in a food-insecure area in Ethiopia. Public Health Nutr 2012;15:1746-54.

44 Bartram J, Cairncross S. Hygiene, sanitation, and water: forgotten foundations of health. PLoS Med 2010;7:e1000367.

45 Velleman Y, Mason E, Graham W, et al. From joint thinking to joint action: a call to action on improving water, sanitation, and hygiene for maternal and newborn health. PLoS Med 2014;11:e1001771.

46 Martorell R. Improved nutrition in the first 1000 days and adult human capital and health. Am J Hum Biol 2017;29. doi:10.1002/ ajhb.22952. [Epub ahead of print: 24 Jan 2017].

47 Victora CG, de Onis M, Hallal PC, et al. Worldwide timing of growth faltering: revisiting implications for interventions. Pediatrics 2010;125:e473-80.

48 Cumming O, Cairncross S. Can water, sanitation and hygiene help eliminate stunting? Current evidence and policy implications. Matern Child Nutr 2016;12(Suppl 1):91-105.

49 Kien VD, Lee H-Y, Nam Y-S, et al. Trends in socioeconomic inequalities in child malnutrition in Vietnam: findings from the multiple indicator cluster surveys, 2000-2011. Glob Health Action 2016;9:29263. 